\title{
Another Phase Plate in the Zoo: Reducing Charging and Optimizing the Design of Electrostatic Phase Plates
}

\author{
Andreas Walter ${ }^{1}$, Siegfried Steltenkamp ${ }^{2}$, Daniel Rhinow ${ }^{1}$ and Werner Kühlbrandt ${ }^{1}$ \\ 1. Max Planck Institute of Biophysics, Department of Structural Biology, Max-von-Laue-Str. 3, D-60438 \\ Frankfurt, Germany \\ 2. caesar Research Center, Ludwig-Erhard-Allee 2, D-53175 Bonn, Germany
}

Phase plates (PPs) enable in-focus transmission electron microscopy (TEM) of frozen-hydrated biological specimens. However, charging of PPs is a problem that has prevented their routine use in TEM of weak-phase objects [1,2]. In theory, electrostatic PPs are superior to thin-film PPs since they do not attenuate the scattered electron beam and allow freely adjustable phase shifts [3]. They consist of multiple layers of conductive and insulating materials, and are thus more prone to charging than thinfilm PPs. Although the origins of PP charging are not fully understood, empirical approaches to reduce charging of PPs have been at least partially successful $[1,4,5]$. However, charging of electrostatic PPs has not been addressed in detail. In this work we address possible origins of charging of Boersch phase plates (BPPs) and suggest a new design that is likely to reduce charging and would be superior to a BPP.

First, we simulated the impact of electrostatic charges of BPPs on phase contrast transfer by performing finite-element calculations with $G m s h / G e t D P$ and integrating the resulting threedimensional potential distribution with MATLAB. We numerically reproduced Fourier patterns that are observed experimentally with charged BPPs and cannot be fitted by a conventional CTF. We showed that surface charges trapped in the vicinity of the BPP central electrode cause circular power spectra, whereas charges along the supporting rods cause a 6-fold distortion. In the simulations, very large phase offsets $(>15 \pi)$ needed to be applied to reproduce the experimentally observed power spectra (Fig. 1a).

We also demonstrated that BPP charging occurs both deeply within the BPP and on its surface. We separated the relative contributions by two main experiments: 1) Monte Carlo simulations with $C A S I N O$ indicated that the penetration depth of $200 \mathrm{keV}$ electrons exceeds the thickness of a $5 \mu \mathrm{m}$ thick $\mathrm{BPP}$, causing inelastic scattering in both the upper and lower insulating $\mathrm{Si}_{3} \mathrm{~N}_{4}$ layer. To address the effect of trapped charges in the insulator we used a FIB instrument to mill ring-shaped cavities around the central electrode on the upper and lower surfaces of the BPP. Subsequently, the cavities were filled with platinum by electron beam-induced deposition to mitigate charges trapped in the insulator in the vicinity of the central electrode. The comparison of Fourier transforms of images of an amorphous carbon film acquired with a BPP before and after fabrication of the charge drains showed that the charge drains reduced the rapid oscillation of Thon ring patterns that are characteristic of charged BPPs. This indicates that electrostatic charges trapped deeply in the BPP are a major factor in the charging of BPPs. 2) In analogy to environmental scanning electron microscopy where positively charged gas molecules in the specimen chamber neutralize negative surface charges trapped by insulators during SEM imaging, we developed a PP goniometer equipped with a gas injection system consisting of metallic nozzles pointing towards the PP chip. To analyse the influence of gas injection on the charging behaviour of BPPs in situ we injected argon in the vacuum of the back-focal plane. The argon flux through the goniometer was kept at flux rates $<0.05 \mathrm{ml} / \mathrm{min}$. The pressure in the specimen chamber increased from $\sim 10^{-7}$ mbar to $\sim 10^{-5}$ mbar. Argon injection affected the Fourier pattern of charged BPPs considerably and immediately, indicating modifications of the BPP surface. Although argon injection did not reduce image artefacts caused by BPPs, the experiments showed that physical and chemical surface properties of PPs are critically important for PP charging, in accordance with other work [4,5]. 
To minimize these charging artifacts and optimize the performance of electrostatic PPs, it is desirable to fabricate electrostatic PPs which expose as little material to the intense unscattered beam as possible. Additionally, an optimal electrostatic PP should impart a homogeneous phase shift to the unscattered beam and have a low cut-on frequency. We propose a new type of electrostatic PP that meets the above requirements and combines the advantages of a single coaxial PP and a BPP. It consists of three free-standing coaxial rods converging in the centre of an aperture (3-fold coaxial PP). To determine the optimum dimensions of the 3-fold coaxial PP and to quantify the homogeneity of the phase shift, we simulated the three-dimensional potential distribution and the induced phase shift with the finite-element method as mentioned above. The simulations shown in Fig. $1 \mathrm{~b}$ indicate that a 3-fold coaxial PP with a rod width $\mathrm{r}$ below $1.2 \mu \mathrm{m}$ is suitable for in-focus imaging of ice-embedded biomolecules. This device combines a rapidly decaying, 3-fold electrostatic field with a nearly constant phase shift of the unscattered beam. This ensures a homogenous and non-directional phase shift of the unscattered beam, which would result in close-to-optimal contrast transfer to near-atomic resolution. The production of a first prototype demonstrates that the free-standing rods are mechanically stable even without the electrode ring and that the fabrication of such a 3 -fold PP is feasible.

[1] A Walter et al, Ultramicroscopy 116 (2012) p. 62-72.

[2] R Danev et al, Ultramicroscopy 109 (2009) p. 312-325.

[3] K Schultheiss et al, Microsc. Microanal. 16 (2010) p. 785-794.

[4] RM Glaser et al, Ultramicroscopy 135 (2013) p. 6-15

[5] M Marko et al, Journal of Structural Biology 184 (2013) p. 237-244
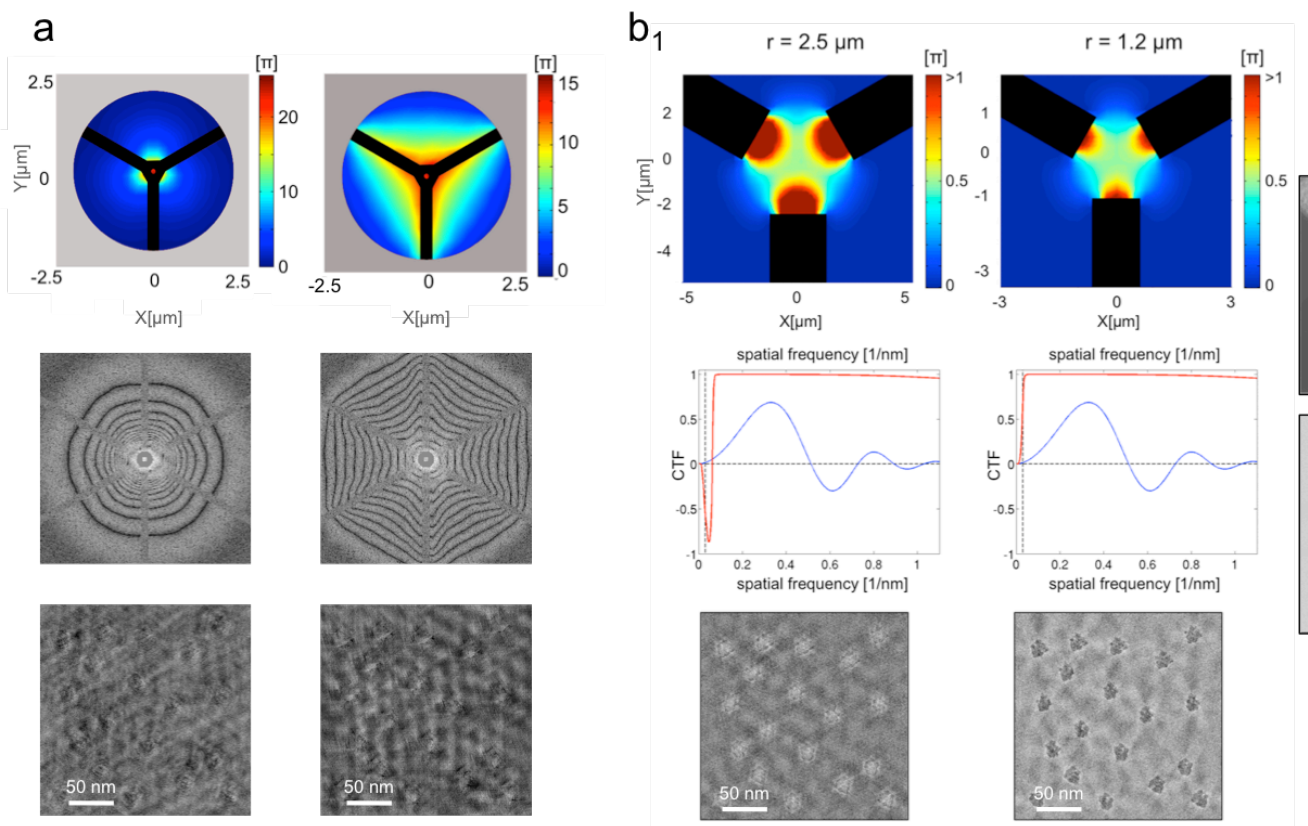

$b_{2}$
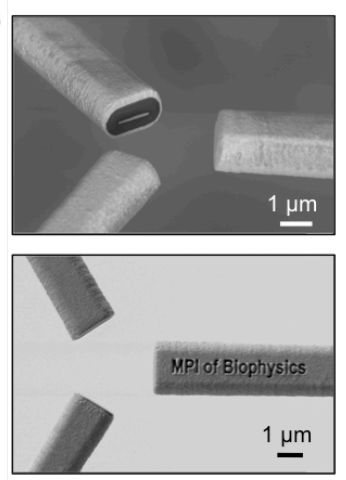

$\underline{\mu m}$

Figure 1. (a) Simulation of phase shifts, power spectra and EM images of ice-embedded ribosomes with a charged BPP nominally in focus. Experimentally observed artifacts in the power spectra were reproduced with positive electrostatic charges homogeneously distributed over the central electrode (1st column) with a charge density $\rho=6^{*} 10^{-5} \mathrm{C} / \mathrm{m}^{2}$ or over the central electrode and along the rods (2nd column) with $\rho=2 * 10^{-5} \mathrm{C} / \mathrm{m}^{2}$. ( $\left.\mathbf{b}_{1}\right)$ Simulation of phase shifts calculated for 3 -fold coaxial PPs with different rod widths, corresponding in-focus CTFs in red (conventionally defocused CTFs in blue), and images of ribosomes. The electrode voltages to produce a $90^{\circ}$ phase shift at the position of the zero beam were $1450 \mathrm{mV}$ for $\mathrm{r}=2.5 \mu \mathrm{m}$ and $700 \mathrm{mV}$ for $\mathrm{r}=1.2 \mu \mathrm{m}$. $\left(\mathbf{b}_{2}\right) \mathrm{SEM}$ images of the first prototype. 